\title{
Preface to the Special Issue on Volunteer Computing and Desktop Grids
}

\author{
Derrick Kondo
}

Published online: 4 November 2009

(C) Springer Science + Business Media B.V. 2009

Desktop Grids and volunteer computing systems (DGVCS's) utilize the free resources available in Intranet or Internet environments for supporting large-scale computation and storage. For over a decade, DGVCS's have been one of the largest and most powerful distributed computing systems in the world, offering a high return on investment for applications from a wide range of scientific domains (including computational biology, climate prediction, and high-energy physics). While DGVCS's sustain up to PetaFLOPS of computing power from hundreds of thousands to millions of resources, fully leveraging the platform's computational power is still a major challenge because of the immense scale, high volatility, and extreme heterogeneity of such systems. This special issue contains journal papers with major contributions that advance the state-of-the-art in the development of scalable, fault-tolerant, and secure DGVCS. In total, we received 26 submissions from which nine were accepted for publication. The topics addressed by these papers include data management, system and application development and experience, scheduling across volatile

D. Kondo $(\varangle)$

INRIA, Grenoble, France

e-mail: derrick.kondo@inria.fr resources, and security for dealing with malicious nodes.

With respect to data management, the authors Xiaosong Ma, Sudharshan Vazhkudai, and Zhe Zhang in their paper titled "Improving Data Availability for Better Access Performance: A Study on Caching Scientific Data on Distributed Desktop Workstations" integrate and evaluate techniques such as prefix caching, collective downloading, and remote partial data recovery for ensuring data availability and low storage consumption.

With respect to system and application development and experience, the authors Peter Kacsuk, Zoltan Balaton, Zoltan Farkas, Gabor Gombas, Jozsef Kovacs and Attila Marosi in their paper titled "SZTAKI Desktop Grid (SZDG): A flexible and scalable desktop Grid system" describe the design and implementation of their middleware for connecting desktop Grids hierarchically, and deploying parameter sweep applications. The authors Raphael Bolze, Frederic Desprez, Kevin Reed, and Viktors Bertis in their paper titled "From Dedicated Grid to Volunteer Grid: Large Scale Execution of a Bioinformatic Application" describe their experience and performance evaluation of running a bioinformatics application on Muscular Dystrophy across volunteered resources as part of the World Community Grid.

With respect to scheduling across volatile resources, the authors Brent Rood and Michael J 
Lewis in their paper titled "Grid Resource Availability Prediction-based Scheduling and Task Replication" apply Markov models in a novel way for predicting resource availability. The authors Eric M. Heien, David P Anderson, and Kenichi Hagihara in their paper titled "Computing Low Latency Batches with Unreliable Workers in Volunteer Computing Environments" develop novel stochastic models and methods for meeting short deadlines of short-lived applications. The authors David Toth and David Finkel in their paper titled "Improving the Productivity of Volunteer Computing by Using the Most Effective Task Retrieval Policies" study the effects of task buffering and division of tasks across single-core and multi-core machines, using different policies of task execution. The authors Michela Taufer, Trilce Estrada, and David P. Anderson in their paper titled "Performance Prediction and Analysis of BOINC Projects: An Empirical Study with EmBOINC describe the design, implementation, and evaluation of a new tool for emulating a real BOINC server and clients.

With respect to security for dealing with malicious nodes, the authors Gheorghe Cosmin Silaghi, Filipe Araujo, Luis Moura Silva, Patricio Domingues, and Alvaro E. Arenas in their paper titled "Defeating Colluding Nodes in Desktop Grid Computing Platforms" use a statistical approach to address the critical problem of ensuring result correctness when malicious clients work together. The authors Kan Watanabe, Masaru Fukushi and Susumu Horiguchi in their paper titled "Optimal Spot-checking for Computation Time Minimization in Volunteer Computing" develop novel techniques that ensure result correctness while minimizing the slowdown due to spot-checking.

To assemble this excellent issue, we gratefully thank the reviewers of the journal submissions. We also thank Peter Kacsuk (editor-in-chief of the Journal of Grid Computing) for his support. 